Kardiologe 2019 $\cdot 13: 38-39$

https://doi.org/10.1007/s12181-018-0291-0

Online publiziert: 6. Dezember 2018

(c) Deutsche Gesellschaft für Kardiologie -

Herz- und Kreislaufforschung e.V.

Published by Springer-Verlag Berlin Heidelberg

- all rights reserved 2018

CrossMark

R. R. Tilz' $\cdot$ K. R. J. Chun ${ }^{2} \cdot$ T. Deneke ${ }^{3} \cdot$ M. Kelm ${ }^{4,9} \cdot$ C. Piorkowski ${ }^{5} \cdot$ P. Sommer ${ }^{6} \cdot$ C. Stellbrink ${ }^{7,9} \cdot$ D. Steven ${ }^{8}$

'Medizinische Klinik II/Kardiologie, Angiologie, Intensivmedizin, Universitäres Herzzentrum Lübeck, Universitätsklinikum Schleswig-Holstein, Lübeck, Deutschland; ${ }^{2}$ Medizinische Klinik III, CCB am Agaplesion Markus Krankenhaus, Frankfurt am Main, Deutschland; ${ }^{3}$ Klinik für Kardiologie/Rhythmologie, Herz- und Gefäß-Klinik Campus Bad Neustadt, Bad Neustadt a. d. Saale, Deutschland; ${ }^{4}$ Klinik für Kardiologie, Pneumologie und Angiologie, Universitätsklinikum Düsseldorf, Düsseldorf, Deutschland; ${ }^{5}$ Klinik für Innere Medizin und Kardiologie, Herzzentrum Dresden GmbH, TU Dresden, Dresden, Deutschland; ${ }^{6}$ Abt. für Rhythmologie, Herzzentrum, Universität Leipzig, Leipzig, Deutschland; ${ }^{7}$ Klinik für Kardiologie und intern. Intensivmedizin, Klinikum Mitte, Städt. Kliniken Bielefeld, Bielefeld, Deutschland;

${ }^{8}$ Abteilung für Elektrophysiologie, Herzzentrum der Universitätsklinik Köln, Köln, Deutschland;

${ }^{9}$ Kommission für Klinische Kardiovaskuläre Medizin, DGK, Düsseldorf, Deutschland

\title{
Erratum zu: Positionspapier der Deutschen Gesellschaft für Kardiologie zur Kardioanalgosedierung
} Fokus auf Eingriffe in der Rhythmologie

\section{Erratum zu:}

Der Kardiologe 2017

https://doi.org/10.1007/s12181-017-

0179-4

Im Abschnitt „Narkotika“, Unterabschnitt „Propofol“ wurde eine fehlerhafte Umrechnung der Medikamentendosis in eine Perfusorlaufrate angegeben. Wir bitten um Beachtung des unten angefügten, korrigierten Abschnitts und bitten den Fehler zu entschuldigen:

Grundsätzlich ist die Einleitung der Sedierung mittels Bolusgabe von einer kontinuierlichen Infusion zu unterscheiden; bei der Bolusgabe werden zumeist 0,5-1 mg/kgKG verabreicht, gefolgt von einer Dauerinfusion mit $1,5-4,5 \mathrm{mg} /$ $\mathrm{kgKG} / \mathrm{h}$. Das heißt: Ein $80 \mathrm{~kg}$-Patient würde einen Bolus von $40 \mathrm{mg}$ erhalten, gefolgt von einer Infusion mit einer Laufrate von $12-36 \mathrm{ml} / \mathrm{h}$ (bei einer Konzentration von $500 \mathrm{mg} / 50 \mathrm{ml}$ Propofol). In der Praxis üblich sind Laufraten von $20-30 \mathrm{ml} / \mathrm{h}$ bei $500 \mathrm{mg} / 50 \mathrm{ml}$, also 200-300 mg/h.

\section{Korrespondenzadresse}

Prof. R. R. Tilz

Medizinische Klinik II/Kardiologie, Angiologie, Intensivmedizin, Universitäres Herzzentrum Lübeck, Universitätsklinikum SchleswigHolstein

Ratzeburger Allee 160, 23538 Lübeck, Deutschland tilz6@hotmail.com

Die Online-Version des Originalartikels ist unter https://doi.org/10.1007/s12181-017-0179-4zu finden. 
Hier steht eine Anzeige.

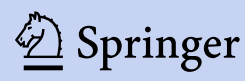

